\title{
Correction to: Effects of oral contraceptives on serum concentrations of adipokines and adiposity indices of women with polycystic ovary syndrome: a randomized controlled trial
}

\author{
M. Amiri ${ }^{1}$ M. Rahmati ${ }^{1,2} \cdot$ M. Hedayati ${ }^{3} \cdot$ F. Nahidi ${ }^{4} \cdot$ F. Ramezani Tehrani ${ }^{1}$
}

Published online: 17 August 2021

(C) Italian Society of Endocrinology (SIE) 2021

\section{Correction to: Journal of Endocrinological Investigation (2021) 44:567-580 \\ https://doi.org/10.1007/s40618-020-01349-8}

The acknowledgement section has been incorrectly published in the original publication. The complete correct acknowledgement is given below.

Authors wish to acknowledge the Research Institute for Endocrine Sciences, Shahid Beheshti University of Medical Sciences for approval of this project as a postdoctoral research fellow project. The authors also thank the Iran National Science Foundation (INSF) for supporting this research project (Grant Number: 97000548).
Publisher's Note Springer Nature remains neutral with regard to jurisdictional claims in published maps and institutional affiliations.

The original article can be found online at https://doi.org/10.1007/ s40618-020-01349-8.

F. Ramezani Tehrani

ramezani@endocrine.ac.ir; framezan@post.harvard.edu

1 Reproductive Endocrinology Research Center, Research Institute for Endocrine Sciences, Shahid Beheshti University of Medical Sciences, No 24, Parvane Street, Yaman Street, Velenjak, Tehran, Iran

2 Department of Epidemiology and Biostatistics, School of Public Health, Tehran University of Medical Sciences, Tehran, Iran

3 Cellular and Molecular Research Center, Research Institute for Endocrine Sciences, Shahid Beheshti University of Medical Sciences, Tehran, Iran

4 Department of Midwifery and Reproductive Health, Faculty of Nursing and Midwifery, Shahid Beheshti University of Medical Sciences, Tehran, Iran 